\title{
Dynamic permeability functions for partially saturated
}

\section{porous media}

\author{
Santiago G. Solazzi ${ }^{1}$, J. Germán Rubino ${ }^{2}$, Damien Jougnot ${ }^{3}$, and Klaus Holliger ${ }^{1,4}$ \\ ${ }^{1}$ Institute of Earth Sciences, University of Lausanne, Lausanne, Switzerland. \\ ${ }^{2}$ CONICET, Centro Atómico Bariloche - CNEA, San Carlos de Bariloche, Argentina. \\ ${ }^{3}$ Sorbonne Université, CNRS, EPHE, UMR 7619, METIS, Paris, France. \\ ${ }^{4}$ School of Earth Sciences, Zhejiang University, Hangzhou, China.
}

\section{SUMMARY}

While the frequency-dependence of permeability under fully saturated conditions has been studied for decades, the corresponding characteristics of partially saturated porous media remain unexplored. Notably, it is not clear whether the use of effective pore fluid approaches under such conditions is valid. To address this issue, we propose a method that allows us to obtain dynamic permeability functions for partially saturated porous media. To this end, we conceptualise the considered pore space as a bundle of capillary tubes of different radii saturated by two immiscible fluid phases. We then solve the Navier-Stokes equations within the pore space and define a capillary pressure-saturation relationship, which permits to obtain saturation- and frequency-dependent effective permeability estimates. The application of this method to a realistic model of an unconsolidated granular sediment demonstrates that dynamic effective permeability functions for wetting and nonwetting fluid phases exhibit distinct characteristics, thus rendering effective pore fluid approaches inadequate. Finally, we explore the capability of the seminal dynamic permeability model developed by Johnson et al. (1987) to account for the effects of partial 
saturation. We find that the frequency scaling proposed by Johnson et al. (1987) prevails in partially saturated scenarios. However, the parameters associated with this model need to be redefined to account for saturation-dependent effects.

Key words: Permeability and porosity, hydrogeophysics, numerical modelling.

\section{INTRODUCTION}

The permeability $\kappa$ is a key hydraulic property of porous media that relates the flow rate per unit area to the fluid pressure drop through Darcy's law and, thus, allows for a comprehensive characterisation of the flow conductance (Bear 1972). In most studies available in the literature, $\kappa$ is considered to be real-valued and frequency-independent. This assumption is valid as long as the fluid flow through the pore space is viscosity-dominated, that is, when the viscous skin depth is greater than the characteristic pore size (Johnson et al. 1987). However, with increasing frequency of the underlying hydraulic pressure variations, inertial effects become more prominent and viscous boundary layers start to develop along the pore walls. This physical process is evidenced as a decrease in the flow rate and a phase shift between the hydraulic pressure variations and the fluid flow. Consequently, the classic formulation of Darcy's law is no longer valid. To circumvent this issue, several investigators have proposed to generalise Darcy's law through the introduction of a complex-valued and frequency-dependent permeability, which is commonly referred to as the dynamic permeability $\kappa(\omega)$, with $\omega$ denoting the angular frequency (e.g., Johnson et al. 1987; Sheng \& Zhou 1988). This concept is of fundamental importance for understanding seismic (e.g., Pride 2005) and seismoelectric signals (e.g., Pride 1994) in fully saturated porous media. To date, dynamic permeability effects in partially saturated porous media have not yet been accounted for in a physically based framework.

Partially saturated geological formations are of preeminent importance in many scientific and applied scenarios in the geosciences, such as exploration and production of hydrocarbons, groundwater management and remediation, and $\mathrm{CO}_{2}$ geosequestration. A better understanding of the frequency dependence of permeability in partially saturated porous media is essential for constraining their hydraulic properties through seismic and/or seismoelectric methods. The absence of a dynamic permeability concept for partially saturated media has led several authors to employ models developed for fully saturated conditions in partially saturated scenarios. Barrière et al. (2012) and Bordes et al. (2015) experimentally explored the behaviour of seismic and seismoelectric signals in partially saturated sand, respectively. They computed $\kappa(\omega)$ based on the seminal model of Johnson et al. (1987) assuming the pores to be saturated by a single fluid phase with effective properties. To date, the validity of approaches of this kind remains unproven and, hence, they might result in erroneous estimations of 
the seismic velocity and attenuation characteristics or the seismoelectric response. Moreover, important theoretical developments in this domain, such as the extension of Biot's (1956a) theory to porous media saturated by two fluid phases (e.g., Lo et al. 2005) and the application of seismoelectric theory to partially saturated media (e.g., Jardani \& Revil 2015) so far inherently rely on the assumption of viscous-dominated flow. The availability of dynamic permeability functions for two-phase flow would permit to extend these theories to the entire frequency band. This possibility would be of significant interest and importance for studying highly permeable partially saturated granular media, which are common in the vadose zone, where dynamic effects are expected to become predominant at relatively low frequencies (e.g., Jouniaux \& Bordes 2012).

In this work, we address this issue by extending the dynamic permeability concept to porous media saturated by two immiscible fluid phases. To this end, we first conceptualise the pore space as being composed of a bundle of capillary tubes of different radii. We solve the Navier-Stokes equations within the pore space to obtain $\kappa(\omega)$ for full saturation. Then, by introducing a capillary pressure-saturation relationship associated with the pore size distribution, we obtain a frequency- and saturation-dependent effective permeability function for each fluid phase. This method is then applied to an unconsolidated sand-type porous medium and compared with seminal end-member-type models. Finally, we use the derived effective permeability functions to explore the capability of the classical model developed by Johnson et al. (1987) to account for the effects of partial saturation.

\section{THEORY}

\subsection{Dynamic permeability for fully saturated porous media}

Let us consider a representative elementary volume (REV) of a fully saturated porous medium in the form of a cylinder of radius $R$ and length $l$. The pore structure is represented by a bundle of capillary tubes, which are aligned with the axis of the cylindrical REV and whose radii $r$ vary from a minimum value $r_{\min }$ to a maximum value $r_{\max }$. The distribution of the pore sizes is such that the number of capillary tubes for radii between $r$ and $r+\mathrm{d} r$ is given by $\mathfrak{f}(r) \mathrm{d} r$. This representation of the pore space can be regarded as a generalisation of the classical model of Kozeny (1927), which provides a good approximation for granular sediments of intermediate to high permeability (e.g., Mavko et al. 2009). Following Johnson et al. (1987), we also consider that the solid matrix is rigid and that the pore space is saturated with an incompressible Newtonian fluid of viscosity $\eta$ and density $\rho$. The fluid can indeed be regarded as incompressible at the pore scale provided that the prevailing acoustic wavelengths in the fluid are much larger than the typical pore size (Johnson et al. 1987; Charlaix et al. 1988; Zhou \& Sheng 1989; Bernabé 1997). We also assume that the fluid flow within the pore space is characterised 
by a small Reynold's number, such that laminar flow prevails (Johnson et al. 1987; Auriault et al. 1985; Smeulders et al. 1992).

The REV is then subjected to an infinitesimal time-harmonic pore fluid pressure difference $\Delta p=$ $\Delta \hat{p} e^{-i \omega t}$ along its axis. The thus resulting volumetric flow within a single capillary tube of radius $r$ can be obtained by solving the incompressible Navier-Stokes equations, under the assumptions described above, which yields (e.g., Johnson et al. 1987)

$$
\hat{q}(r, \omega)=-\frac{\pi r^{2}}{\eta k^{2}}\left[\frac{2}{k r} \frac{J_{1}(k r)}{J_{0}(k r)}-1\right] \frac{\Delta \hat{p}}{l},
$$

where $k^{2}=i \omega \rho / \eta$ and $J_{\zeta}$ are Bessel functions of the first kind of order $\zeta=0,1$. The harmonic term $e^{-i \omega t}$ has been dropped for ease of notation.

The volumetric flow rate $\hat{Q}$ at the REV scale can be obtained by integrating eq. (1) over the entire range of pore sizes

$$
\hat{Q}=\int_{r_{\min }}^{r_{\max }} \hat{q}(r, \omega) \mathfrak{f}(r) \mathrm{d} r
$$

Correspondingly, the effective Darcy velocity at the REV scale is given by $\hat{v}=\hat{Q} / \pi R^{2}$. The dynamic permeability can therefore be computed by considering Darcy's relationship between the fluid flow and the prevailing pressure gradient, which yields

$$
\kappa(\omega)=\frac{1}{R^{2} k^{2}} \int_{r_{\min }}^{r_{\max }}\left[\frac{2}{k r} \frac{J_{1}(k r)}{J_{0}(k r)}-1\right] r^{2} \mathfrak{f}(r) \mathrm{d} r .
$$

This equation can be solved numerically once $\mathfrak{f}(r)$ as well as $r_{\min }$ and $r_{\max }$ have been defined.

It is important to remark here that the transition from viscous- to inertia-dominated flow occurs at the so-called critical angular frequency $\omega_{\mathrm{c}}$, for which the viscous skin depth $\delta=\sqrt{2 \eta / \rho \omega}$ becomes comparable to the radii of the largest saturated pores (Johnson et al. 1987). Then, the critical angular frequency satisfies

$$
\omega_{\mathrm{c}} \simeq \frac{2 \eta}{\rho \tilde{r}^{2}}
$$

where $\tilde{r}$ is a characteristic radius representative of the largest saturated pores, which, for the considered pore size distribution is controlled by $r_{\max }$. In the following, we present a model to extend eq. (3) to partially saturated media.

\subsection{Dynamic effective permeability functions for partially saturated porous media}

Whenever a porous rock is saturated by two immiscible fluids, only a fraction of the corresponding medium contributes to the flow of each fluid phase and, thus, corresponding effective permeabilities need to be defined. The classical two-phase flow formulation of Darcy's law is given by (e.g., Bear 
1972)

$$
\mathbf{v}_{\mathrm{w}}=-\frac{\kappa_{\mathrm{w}}^{\mathrm{eff}}}{\eta_{\mathrm{w}}} \nabla p_{\mathrm{w}}, \quad \mathbf{v}_{\mathrm{n}}=-\frac{\kappa_{\mathrm{n}}^{\mathrm{eff}}}{\eta_{\mathrm{n}}} \nabla p_{\mathrm{n}}
$$

where the subscripts $\mathrm{w}$ and $\mathrm{n}$ refer to the wetting and non-wetting fluid phases saturating the pores, respectively. In eq. (5), $\mathbf{v}_{\alpha}$ is Darcy's velocity, $\kappa_{\alpha}^{\text {eff }}$ is the effective permeability, $p_{\alpha}$ is the fluid pressure, and $\eta_{\alpha}$ is the viscosity, with $\alpha=\mathrm{w}, \mathrm{n}$ denoting the fluid phase.

The capillary pressure $p_{\mathrm{c}}$ of a partially saturated cylindrical tube of radius $r_{\mathrm{p}}$ is given by the Young-Laplace equation (Bear 1972)

$$
p_{\mathrm{c}}=\frac{2 \gamma \cos (\beta)}{r_{\mathrm{p}}},
$$

where $\gamma$ denotes the interfacial tension between the two immiscible fluid phases and $\beta$ is the contact angle. The capillary pressure is related to the saturation state of the medium (e.g., Brooks \& Corey 1964). Indeed, if we consider the same pore space topology as in the previous section and assume a given state of capillary pressure equilibrium, all pores with radii $r \leq r_{\mathrm{p}}\left(p_{\mathrm{c}}\right)=\frac{2 \gamma \cos (\beta)}{p_{\mathrm{c}}}$ are saturated by the wetting phase. Conversely, the pores with $r>r_{\mathrm{p}}\left(p_{\mathrm{c}}\right)$ are saturated by the non-wetting phase (e.g., Mualem 1976). Then, the effective wetting phase saturation $S_{\mathrm{ew}}\left(p_{\mathrm{c}}\right)$ responds to (e.g., Blunt 2017)

$$
S_{\text {ew }}\left(p_{\mathrm{c}}\right)=\frac{\int_{r_{\min }}^{r_{\mathrm{p}}\left(p_{\mathrm{c}}\right)} r^{2} \mathfrak{f}(r) \mathrm{d} r}{\int_{r_{\min }}^{r_{\max }} r^{2} \mathfrak{f}(r) \mathrm{d} r}, \text { for } \quad p_{\mathrm{c}, \min } \leq p_{\mathrm{c}} \leq p_{\mathrm{c}, \max },
$$

with $p_{\mathrm{c}, \min }=2 \gamma \cos \beta / r_{\max }$ and $p_{\mathrm{c}, \max }=2 \gamma \cos \beta / r_{\min }$. If $p_{\mathrm{c}}<p_{\mathrm{c}, \min }$ we have $S_{\mathrm{ew}}=1$ and, conversely, if $p_{\mathrm{c}}>p_{\mathrm{c}, \max }$ we have $S_{\mathrm{ew}}=0$. Eq. (7) is based on the assumption that the pore fluid distribution is achieved through a flow process in which capillary forces are predominant. Hence, each fluid phase saturates a particular sub-set of the probed porous medium, which, in turn, is determined by the capillary pressure state (Blunt 2017). The effective $S_{\text {ew }}$ and total $S_{\mathrm{w}}$ saturations of the medium are related by $S_{\mathrm{w}}=S_{\mathrm{ew}}\left(1-S_{\mathrm{wr}}-S_{\mathrm{nr}}\right)+S_{\mathrm{wr}}$, where $S_{\mathrm{wr}}$ and $S_{\mathrm{nr}}$ denote the residual saturations for the wetting and non-wetting phases, respectively.

The effective volumetric flow rates for the wetting and non-wetting fluid phases can be obtained by integrating eq. (2) between $r_{\min }$ and $r_{\mathrm{p}}\left(p_{\mathrm{c}}\right)$ and between $r_{\mathrm{p}}\left(p_{\mathrm{c}}\right)$ and $r_{\max }$, respectively. Then, employing eq. (5), we define the frequency-dependent dynamic effective permeabilities for the wetting and non-wetting phases as

$$
\begin{aligned}
\kappa_{\mathrm{w}}^{\mathrm{eff}}\left(p_{\mathrm{c}}, \omega\right) & =\frac{1}{R^{2} k_{\mathrm{w}}^{2}} \int_{r_{\min }}^{r_{\mathrm{p}}\left(p_{\mathrm{c}}\right)}\left[\frac{2}{k_{\mathrm{w}} r} \frac{J_{1}\left(k_{\mathrm{w}} r\right)}{J_{0}\left(k_{\mathrm{w}} r\right)}-1\right] r^{2} \mathfrak{f}(r) \mathrm{d} r, \\
\kappa_{\mathrm{n}}^{\mathrm{eff}}\left(p_{\mathrm{c}}, \omega\right) & =\frac{1}{R^{2} k_{\mathrm{n}}^{2}} \int_{r_{\mathrm{p}}\left(p_{\mathrm{c}}\right)}^{r_{\max }}\left[\frac{2}{k_{\mathrm{n}} r} \frac{J_{1}\left(k_{\mathrm{n}} r\right)}{J_{0}\left(k_{\mathrm{n}} r\right)}-1\right] r^{2} \mathfrak{f}(r) \mathrm{d} r,
\end{aligned}
$$

where $k_{\alpha}^{2}=i \omega \rho_{\alpha} / \eta_{\alpha}$, with $\alpha=\mathrm{w}, \mathrm{n}$.

Eqs (8) and (9) are the central methodological result of this paper. Together with eq. (7), they 
provide a dynamic effective permeability-saturation relationship for each fluid phase of a partially saturated porous medium. In the low-frequency limit, these expressions converge to their Poiseuilletype counterparts (e.g., Blunt 2017)

$$
\begin{aligned}
\kappa_{\mathrm{w} 0}^{\mathrm{eff}}\left(p_{\mathrm{c}}\right) & =\frac{1}{8 R^{2}} \int_{r_{\min }}^{r_{\mathrm{p}}\left(p_{\mathrm{c}}\right)} r^{4} \mathfrak{f}(r) \mathrm{d} r, \\
\kappa_{\mathrm{n} 0}^{\mathrm{eff}}\left(p_{\mathrm{c}}\right) & =\frac{1}{8 R^{2}} \int_{r_{\mathrm{p}}\left(p_{\mathrm{c}}\right)}^{r_{\max }} r^{4} \mathfrak{f}(r) \mathrm{d} r .
\end{aligned}
$$

A particular strength of the proposed model is that it is designed to allow for virtually arbitrary pore size distributions $\mathfrak{f}(r)$. Moreover, $\mathfrak{f}(r)$ can be inferred from the hydrodynamic characteristic curves of any porous medium, using either the capillary pressure-saturation or relative permeability-saturation relationships (Jougnot et al. 2012). Finally, eqs (8) and (9) can be readily modified to account for a tortuosity factor associated with the non-alignment of the capillary tubes with the sample axis (e.g., Bedford et al. 1984).

Due to the irreversibility of multiphase flow dynamics, the fluid distribution in porous media depends on the flow history (e.g., Lenormand 1990). Such hysteretic effects are, however, beyond the scope of this work, which seeks to elucidate the foundations of dynamic permeability in partially saturated porous media.

\section{NUMERICAL CASE STUDY}

To explore the characteristics of the dynamic effective permeability model for partial saturation given by eqs (8) and (9), we consider a cylindrical REV of porous material with a radius $R$ of $2.4 \mathrm{~cm}$. The pore space topology is assumed to be characterised by a fractal distribution of capillary tubes given by $\mathfrak{f}(r)=D R^{D} r^{-D-1}$, where $1<D<2$ is the fractal dimension (e.g., Soldi et al. 2017). Based on eq. (7), we obtain

$$
S_{\text {ew }}\left(p_{\mathrm{c}}\right)=\frac{p_{\mathrm{c}}^{D-2}-p_{\mathrm{c}, \text { max }}^{D-2}}{p_{\mathrm{c}, \text { min }}^{D-2}-p_{\mathrm{c}, \text { max }}^{D-2}}, \text { with } p_{\mathrm{c}, \text { min }} \leq p_{\mathrm{c}} \leq p_{\mathrm{c}, \text { max }} \text {. }
$$

Following Brakensiek \& Rawls (1992), we use $D=1.41$, which is characteristic of unconsolidated sandy materials. The maximum and minimum radii are taken as $r_{\max }=111 \mu \mathrm{m}$ and $r_{\min }=$ $10^{-2} r_{\max }$, respectively (Assouline \& Or 2008; Yu 2008). These parameters result in a low-frequency permeability of $\kappa_{0}=\int_{r_{\min }}^{r_{\max }} r^{4} \mathfrak{f}(r) \mathrm{d} r /\left(8 R^{2}\right)=35.6 \mathrm{D}$ (e.g., Blunt 2017), which is typical of unconsolidated sand (e.g., Schön 2015). The wetting fluid is assumed to be water, with $\rho_{\mathrm{w}}=1000 \mathrm{~kg} / \mathrm{m}^{3}$ and $\eta_{\mathrm{w}}=0.001$ Pa.s, while the non-wetting fluid is air, with $\rho_{\mathrm{n}}=1 \mathrm{~kg} / \mathrm{m}^{3}$ and $\eta_{\mathrm{n}}=2 \times 10^{-5}$ Pa.s. The interfacial tension and the contact angle are taken as $\gamma=0.072 \mathrm{~N} / \mathrm{m}$ and $\beta=0^{\circ}$, respectively (Vargaftik et al. 1983). To allow for a direct comparison of the proposed model with that of John- 


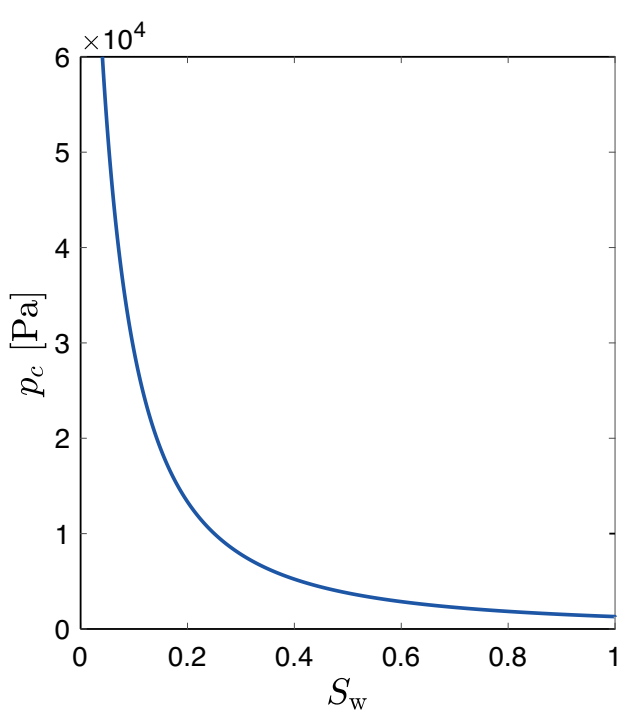

Figure 1. Capillary pressure $p_{\mathrm{c}}$ as a function of water saturation $S_{\mathrm{w}}$ for the considered porous medium.

son et al. (1987), which has been developed for fully saturated conditions, we assume that residual saturations are negligible, that is, $S_{\mathrm{w}}=S_{\text {ew }}$ (Fig. 1).

Our results demonstrate that, regardless of the frequency, the effective permeability of water decreases with decreasing water saturation $S_{\mathrm{w}}$ (Figs 2a and 2b), which is due to the fact that progressively smaller portions of the medium contribute to the flow of this fluid phase. Evidently, the effective water permeability is null for $S_{\mathrm{w}}=0$. We also observe that, irrespective of the saturation state of the sample, the absolute value of $\kappa_{\mathrm{w}}^{\mathrm{eff}}$ is fairly stable for low frequencies and that, for frequencies greater than a given threshold value, it decreases (Fig. 2a). This behaviour evidences the development of viscous boundary layers within the pores, thus indicating that the threshold value is associated with the location of the critical frequency $f_{\mathrm{c}}=\omega_{\mathrm{c}} / 2 \pi$ (Johnson et al. 1987). Interestingly, the value of $f_{\mathrm{c}}$ moves to higher frequencies as the medium is desaturated. The reason for this is that the characteristic size of the largest water-saturated pores $\tilde{r}$ involved in eq. (4) is indeed controlled by $r_{\mathrm{p}}$ due to the wetting properties of water, which decreases for lower water saturations. While the phase of $\kappa_{\mathrm{w}}^{\mathrm{eff}}$ is null for low frequencies, it increases with frequency together with the inertial forces within the pore space, thus evidencing the out-of-phase movement of the water flow with respect to the pressure forcing (Fig. 2b). The inflections in the phase of $\kappa_{\mathrm{w}}^{\mathrm{eff}}$ move to higher frequencies as the medium is desaturated. Finally, we plot the classical model of Johnson et al. (1987) for full saturation

$$
k^{\mathrm{J}}(\omega)=k_{0}\left(\sqrt{1-\frac{i M}{2} \frac{\omega}{\omega_{\mathrm{c}}}}-i \frac{\omega}{\omega_{\mathrm{c}}}\right)^{-1}
$$



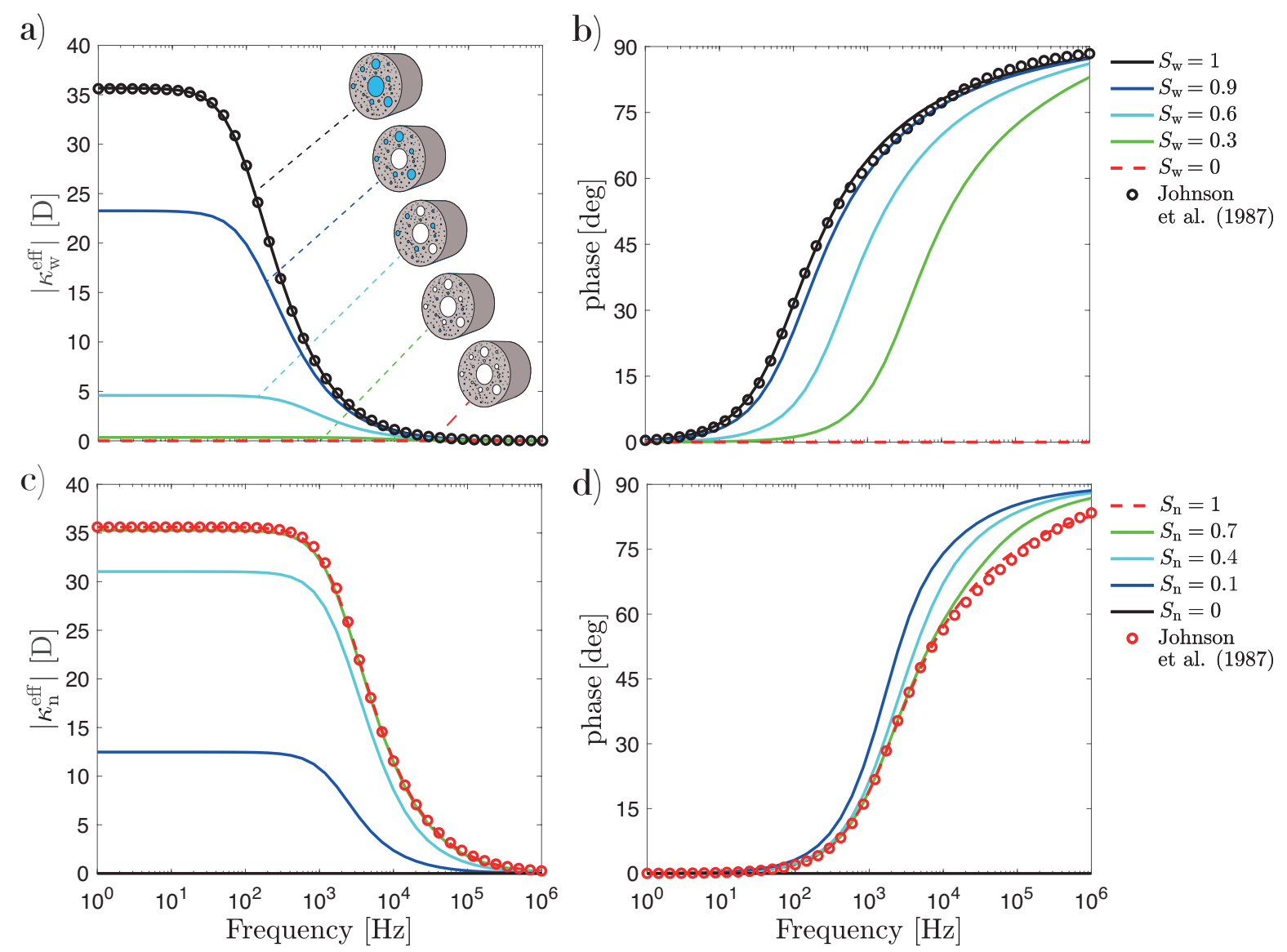

Figure 2. Absolute values and phases of $(\mathrm{a}, \mathrm{b}) \kappa_{\mathrm{w}}^{\mathrm{eff}}$ and (c, d) $\kappa_{\mathrm{n}}^{\text {eff }}$ as functions of frequency for different saturations. Sketches within panel (a) illustrate the different saturation states of the considered REV with waterand air-saturated pores denoted in blue and white, respectively.

where the critical angular frequency $\omega_{c}$ and the $M$ parameter are given by

$$
\omega_{\mathrm{c}}=\frac{\phi \eta_{\mathrm{w}}}{\kappa_{0} \rho_{\mathrm{w}} \tau}, \quad M=\frac{8 \tau \kappa_{0}}{\Lambda^{2} \phi} .
$$

We take the tortuosity $\tau$ equal to 1 , in agreement with the proposed model, and the porosity as $\phi=\int_{r_{\min }}^{r_{\max }} r^{2} \mathfrak{f}(r) \mathrm{d} r / R^{2}$ (e.g., Blunt 2017). For a bundle of non-intersecting tubes, the characteristic dynamic surface-to-volume ratio $\Lambda$ is given by $\Lambda=\int_{r_{\min }}^{r_{\max }} r^{2} \mathfrak{f}(r) \mathrm{d} r / \int_{r_{\min }}^{r_{\max }} r \mathfrak{f}(r) \mathrm{d} r$ (Johnson et al. 1987), which, in this case, results in 0.116 $r_{\max }$. However, according to Achdou \& Avellaneda (1992), this expression tends to overestimate the contribution of smaller pores, which are predominant in the considered fractal distribution. In this context, we note that $\Lambda=0.17 \cdot r_{\max }$ provides the best approximation of the dynamic permeability behaviour with respect to the $L_{2}$-norm. The proposed model, given by eq. (8), is indeed in excellent agreement with the frequency-dependent behaviour predicted by the model of Johnson et al. (1987) at full water saturation (Figs 2a and 2b). The characteristics of 
the model of Johnson et al. (1987) for partially saturated porous media will be further discussed in section 4.

The absolute value and phase of $\kappa_{\mathrm{n}}^{\mathrm{eff}}$ show a similar behaviour with $S_{\mathrm{n}}$ as $\kappa_{\mathrm{w}}^{\text {eff }}$ does with $S_{\mathrm{w}}$ (Figs $2 \mathrm{c}$ and $2 \mathrm{~d}$ ). The critical frequency $f_{\mathrm{c}}$ for the non-wetting phase is located at higher frequencies than that of the wetting phase due to the larger kinematic viscosity $\nu=\eta / \rho$ of air compared to water. $f_{\mathrm{c}}$ appears to be fairly stable with regard to $S_{\mathrm{n}}$, because the characteristic size $\tilde{r}$ of the largest airinvaded pores is controlled by $r_{\max }$ due to the non-wetting properties of air. The characteristic pore size tends to determine when viscous boundary layers first form within the pore space (eq. 4) and, thus, little change is expected with regard to the critical frequency with increasing air saturation. This observation is important as it illustrates that wetting and non-wetting fluid phases have fundamentally different dynamic effective permeability behaviours with respect to the saturation state. Finally, we observe that, also in this case, there is good agreement between the proposed model (eq. 9) and that of Johnson et al. (1987) for full air saturation. Please note that the dynamic effects prevail at seismic and sonic frequencies (Fig. 2).

We calculate the low-frequency effective permeabilities $\kappa_{\mathrm{w} 0}^{\mathrm{eff}}$ and $\kappa_{\mathrm{n} 0}^{\mathrm{eff}}$ according to equations (10) and (11), which, for the considered fractal distribution, are given by

$$
\begin{aligned}
& \kappa_{\mathrm{w} 0}^{\mathrm{eff}}\left(p_{\mathrm{c}}\right)=\frac{D}{8 R^{2-D}(4-D)}\left[r_{\mathrm{p}}\left(p_{\mathrm{c}}\right)^{4-D}-r_{\min }^{4-D}\right], \\
& \kappa_{\mathrm{n} 0}^{\mathrm{eff}}\left(p_{\mathrm{c}}\right)=\frac{D}{8 R^{2-D}(4-D)}\left[r_{\max }^{4-D}-r_{\mathrm{p}}\left(p_{\mathrm{c}}\right)^{4-D}\right],
\end{aligned}
$$

and compare them to the $\kappa_{\mathrm{w}}^{\text {eff }}$ and $\kappa_{\mathrm{n}}^{\text {eff }}$ at $10 \mathrm{~Hz}, 10^{3} \mathrm{~Hz}, 10^{4} \mathrm{~Hz}$, and $10^{5} \mathrm{~Hz}$ (Fig. 3). The absolute value of $\kappa_{\mathrm{w}}^{\text {eff }}$ increases with $S_{\mathrm{w}}$ (Fig. 3a). For frequencies below $f_{\mathrm{c}}$, such as $f=10 \mathrm{~Hz}$, eq. (15) agrees with the absolute values of $\kappa_{\mathrm{w}}^{\mathrm{eff}}$, which implies that, in the low-frequency range, the dynamic effective permeability of the wetting phase is consistent with its corresponding Poiseuille-type model, while, above $f_{\mathrm{c}}$, the absolute value of $\kappa_{\mathrm{w}}^{\mathrm{eff}}$ decreases with increasing frequency (Figs $2 \mathrm{a}$ and $3 \mathrm{a}$ ). The phase of $\kappa_{\mathrm{w}}^{\mathrm{eff}}$ (Fig. 3b) increases with saturation and assumes smaller values for decreasing frequencies. At the low-frequency limit, the phase is, as expected, virtually null. The absolute value of $\kappa_{\mathrm{n}}^{\text {eff }}$ decreases with increasing values of $S_{\mathrm{w}}$ (Fig. 3c). The behaviour of the absolute value of $\kappa_{\mathrm{n}}^{\mathrm{eff}}$ in the low-frequency range for air reproduces its corresponding Poiseuille-type behaviour as well as the wetting fluid does. The discrepancy between the absolute value of $\kappa_{\mathrm{n}}^{\mathrm{eff}}$ and $\kappa_{\mathrm{n} 0}^{\mathrm{eff}}$ increases with increasing frequency. The phase of $\kappa_{\mathrm{n}}^{\mathrm{eff}}$ does not vary significantly with saturation for the non-wetting fluid (Fig. 2d). Hence, the phase of $\kappa_{\mathrm{n}}^{\mathrm{eff}}$ remains fairly stable with saturation, showing increasing values with frequency.

The relationship between the angular critical frequency $\omega_{\mathrm{c}}$ and the water saturation $S_{\mathrm{w}}$ is of crucial importance as it determines the frequency above which dynamic effects need to be considered for a given partially saturated material (Fig. 4). Following Bernabé (1997), the values of $\omega_{\mathrm{c}}\left(S_{\mathrm{w}}\right)$ analyzed 

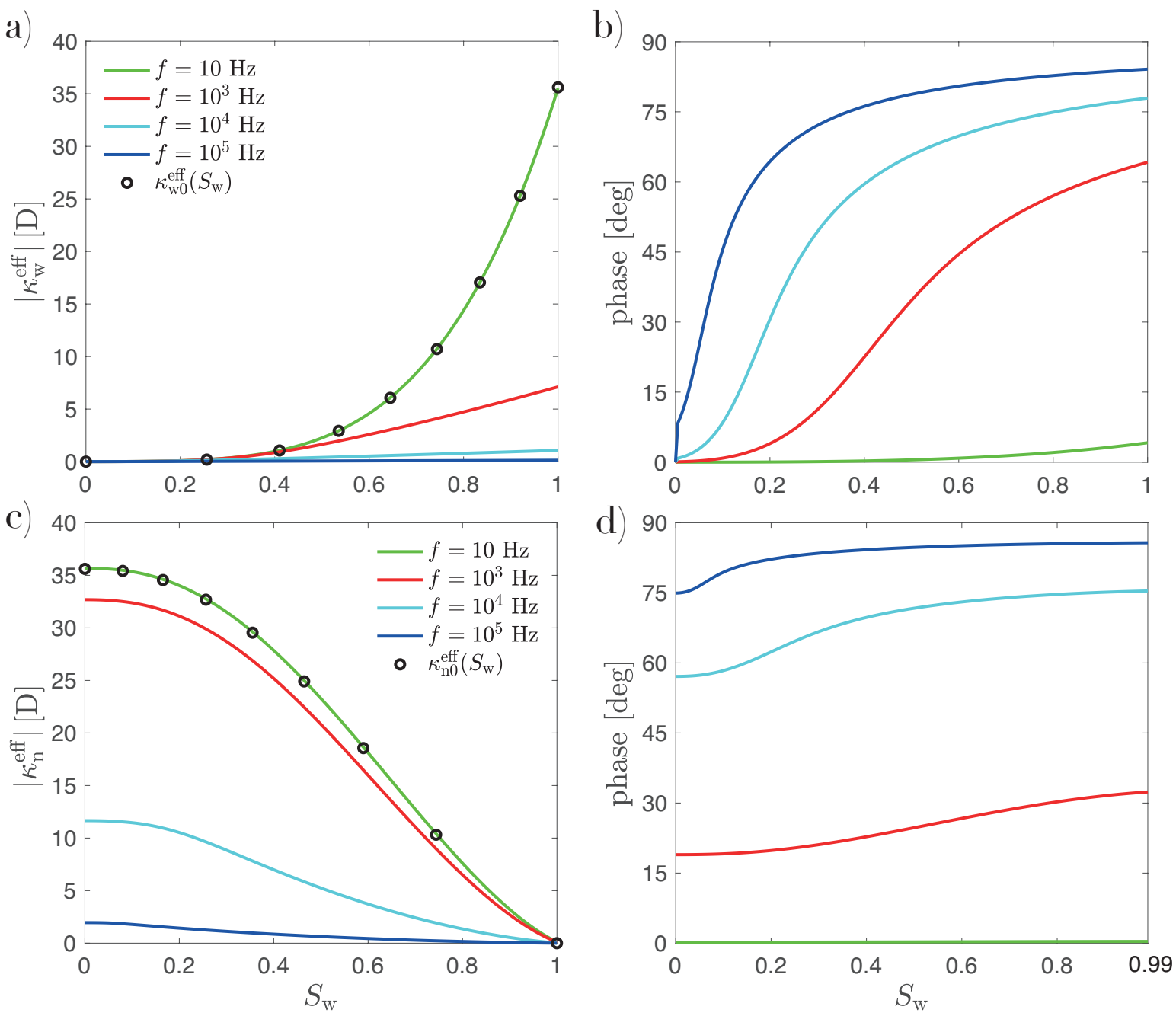

Figure 3. Absolute values and phases of $(\mathrm{a}, \mathrm{b}) \kappa_{\mathrm{w}}^{\mathrm{eff}}$ and $(\mathrm{c}, \mathrm{d}) \kappa_{\mathrm{n}}^{\mathrm{eff}}$ as functions of water saturation $S_{\mathrm{w}}$ for different frequencies.

here correspond to the angular frequencies at which $\Im\left\{\kappa_{\mathrm{w}}^{\mathrm{eff}}\left(\omega, S_{\mathrm{w}}\right)\right\}$ and $\Im\left\{\kappa_{\mathrm{n}}^{\text {eff }}\left(\omega, S_{\mathrm{w}}\right)\right\}$ assume their maximum values. We have normalized these values with respect to their water-saturated end-member value $\omega_{\mathrm{c}}\left(S_{\mathrm{w}}=1\right)$. We find that $\omega_{\mathrm{c}}$ decreases with $S_{\mathrm{w}}$ for water while it remains fairly constant for air (Fig. 4a). Furthermore, $\omega_{\mathrm{c}}$ increases with $p_{\mathrm{c}}$ for water while it again remains fairly constant for air (Fig. 4b). This behaviour can be understood taking into account that the value of the characteristic pore size $\tilde{r}$ involved in eq. (4) is controlled by $r_{\mathrm{p}}\left(p_{\mathrm{c}}\right)$ for the wetting phase and by $r_{\max }$ for the non-wetting phase. These results disagree with those obtained by Barrière et al. (2012) and Bordes et al. (2015), where a single pore fluid phase with the following effective properties

$$
\begin{aligned}
& \rho_{\mathrm{eff}}=\rho_{\mathrm{w}} S_{\mathrm{w}}+\rho_{\mathrm{n}}\left(1-S_{\mathrm{w}}\right), \\
& \eta_{\mathrm{eff}}=\eta_{\mathrm{n}}\left(\frac{\eta_{\mathrm{w}}}{\eta_{\mathrm{n}}}\right)^{S_{\mathrm{w}}},
\end{aligned}
$$



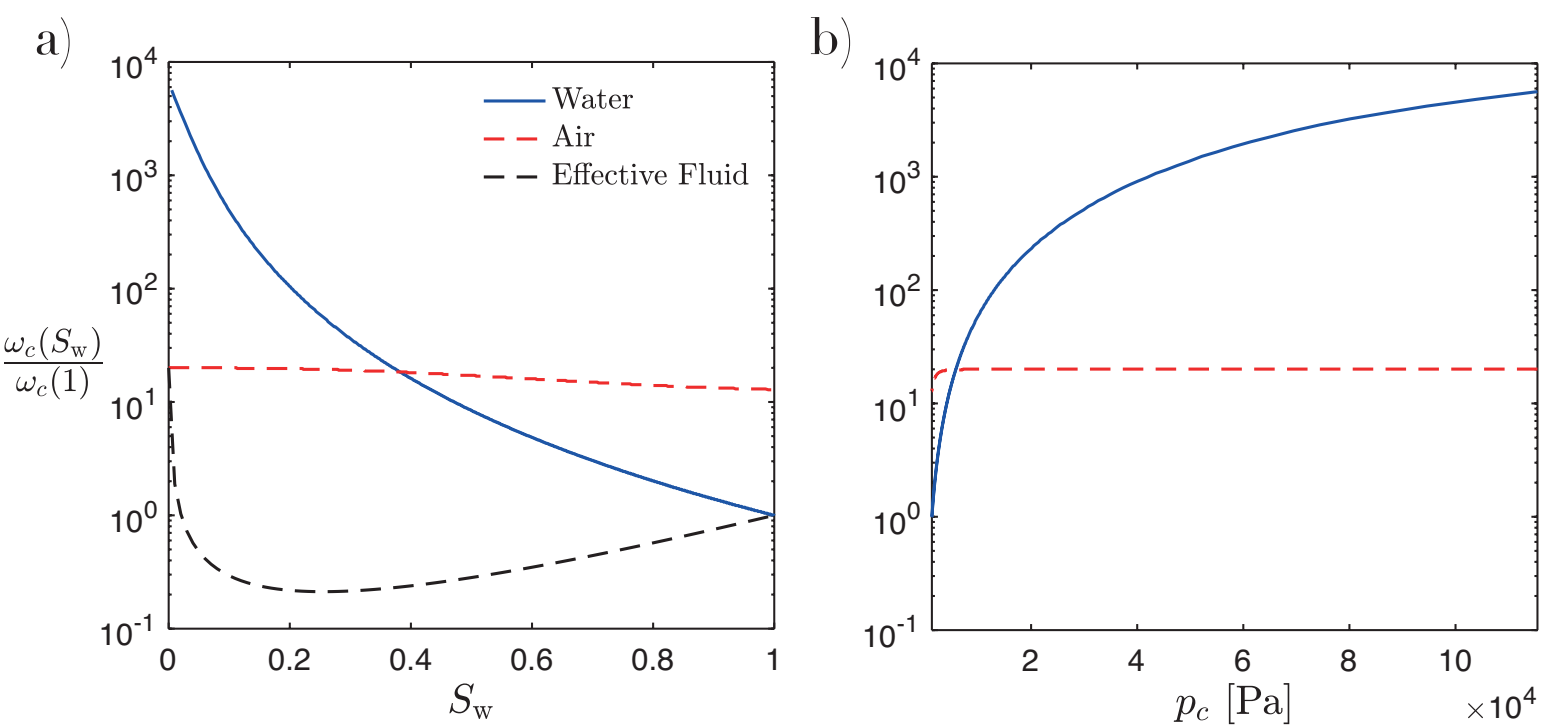

Figure 4. Normalized critical frequency for water (blue solid lines) and air (red dashed lines) as a function of (a) water saturation $S_{\mathrm{w}}$ and (b) capillary pressure $p_{\mathrm{c}}$. For comparison, we illustrate in (a) the behaviour of the critical frequency using an effective fluid (black dashed lines), computed following equation (14).

is considered to approximate the behaviour of a partially saturated medium using the model of Johnson et al. (1987). The results of these studies erroneously suggest that $\omega_{\mathrm{c}}$ decreases with $S_{\mathrm{w}}$ for saturations below $\sim 0.2$ and otherwise continuously increases with $S_{\mathrm{w}}$ (Fig. $4 \mathrm{a}$ ), which is a consequence of considering eqs (17) and (18) when computing $\omega_{\mathrm{c}}$ using eq. (14). These results illustrate that considering an effective fluid to compute the dynamic permeability of a partially saturated medium is physically incorrect, which is of critical importance when modelling the seismic and/or seismoelectric signatures of partially saturated media using Biot's equations of poroelasticity (Biot 1956a,b).

\section{PERFORMANCE OF “JOHNSON'S MODEL” IN PARTIALLY SATURATED MEDIA}

The model of Johnson et al. (1987) is arguably the most widely used approach to account for inertia effects on the permeability in the context of seismic and seismoelectric studies of porous media. The reason for this is twofold: (1) The derived expression is relatively simple and depends on measurable pore space parameters; (2) the model has been proven to be viable and robust when compared with experimental measurements performed on synthetic aggregates (e.g., Charlaix et al. 1988; Smeulders et al. 1992). However, deviations from the behavior predicted by Johnson et al. (1987) have been observed when the pore space contains constrictions (e.g., Pride et al. 1993) and/or when the pore size distribution is characterized by a large amount of small pores, as is indeed the case for the fractal pore size distribution considered in the previous section (e.g., Achdou \& Avellaneda 1992; Bernabé 1997). In the latter case, the dynamic surface-to-volume ratio does not provide with a good fit in the 
context of Johnson et al. (1987) model. Conversely, the frequency scaling proposed by Johnson et al. (1987) is sound and the predicted characteristics appear to be representative of partially saturated conditions (Fig 2). In this section, we therefore explore if the frequency scaling proposed by Johnson et al. (1987) can be employed to represent the behavior of effective dynamic permeability functions in partially saturated porous media.

Firstly, we need to account for the variation of the parameters involved in the model of Johnson et al. (1987) with saturation. Generalizing eqs (13) and (14), we propose

$$
k_{\alpha}^{\mathrm{J}, *}\left(S_{\alpha}, \omega\right)=\kappa_{\alpha 0}^{\mathrm{eff}}\left(S_{\alpha}\right)\left(\sqrt{1-\frac{i M_{\alpha}\left(S_{\alpha}\right)}{2} \frac{\omega}{\omega_{\mathrm{c}, \alpha}\left(S_{\alpha}\right)}}-i \frac{\omega}{\omega_{\mathrm{c}, \alpha}\left(S_{\alpha}\right)}\right)^{-1}, \quad \text { with } \alpha=\mathrm{w}, \mathrm{n},
$$

where

$$
\omega_{\mathrm{c}, \alpha}\left(S_{\alpha}\right)=\frac{S_{\alpha} \phi \eta_{\alpha}}{\kappa_{\alpha 0}^{\mathrm{eff}}\left(S_{\alpha}\right) \rho_{\alpha} \tau_{\alpha}\left(S_{\alpha}\right)}, \quad M_{\alpha}\left(S_{\alpha}\right)=\frac{8 \tau\left(S_{\alpha}\right) \kappa_{\alpha 0}^{\mathrm{eff}}\left(S_{\alpha}\right)}{\Lambda_{\alpha}^{2}\left(S_{\alpha}\right) S_{\alpha} \phi}
$$

In these equations, $\kappa_{\alpha 0}^{\mathrm{eff}}\left(S_{\mathrm{w}}\right)$ denotes the effective permeability of phase $\alpha$ in the low-frequency range. In addition, the term $S_{\alpha} \phi$ in eq. (20) permits to account for the fraction of the medium invaded by each pore fluid phase. Finally, in agreement with the proposed pore space topology, we set $\tau_{\alpha} \equiv 1$ and assume $\Lambda_{\alpha}$ to be a function of the saturation for each pore fluid phase.

For each saturation state, we perform an exhaustive search of the parameters $\Lambda_{\mathrm{w}}$ and $\Lambda_{\mathrm{n}}$ in eq. (19) that provide the best fit to the behavior predicted by eqs (8) and (9). We consider that the porous medium has the same properties as those employed in the last section and, thus, we rely on eqs (15) and (16) to compute the parameters $\kappa_{\mathrm{w} 0}^{\mathrm{eff}}\left(S_{\mathrm{w}}\right)$ and $\kappa_{\mathrm{n} 0}^{\mathrm{eff}}\left(S_{\mathrm{n}}\right)$.

The response predicted by eq. (19) provides an excellent representation of the effective relative permeabilities for the probed medium (Fig. 5). This consistency indicates that the frequency scaling proposed by Johnson et al. (1987) for full saturation prevails under partially saturated conditions. More importantly, the characteristic frequency defined in eq. (20) correctly represents the viscous-to-inertia transition frequency for partially saturated conditions and, thus, should be considered instead of the effective fluid properties in its monosaturated counterpart (eq. 14).

The values of $\Lambda$ that provide the best fit for each saturation state are different for the effective wetting and non-wetting dynamic permeability functions (Figs 6a and 6b). $\Lambda_{\mathrm{w}}$ increases with $S_{\mathrm{w}}$ (Fig. 6a). Taking into account that $\Lambda_{\mathrm{w}}$ is indicative of a characteristic pore scale involved in the flow of the wetting fluid, this behavior is expected, as larger pores are invaded by water for increasing saturation values. Interestingly, similar relationships between $\Lambda$ and $S_{\mathrm{w}}$ have been observed by Maineult et al. (2017) who studied the spectral induced polarization of partially saturated random tube networks. In the case of the non-wetting fluid phase, $\Lambda_{\mathrm{n}}$ decreases with air saturation $S_{\mathrm{n}}$ (Fig. 6b). This behavior is expected, as when the medium is increasingly saturated with air, the non-wetting phase invades 

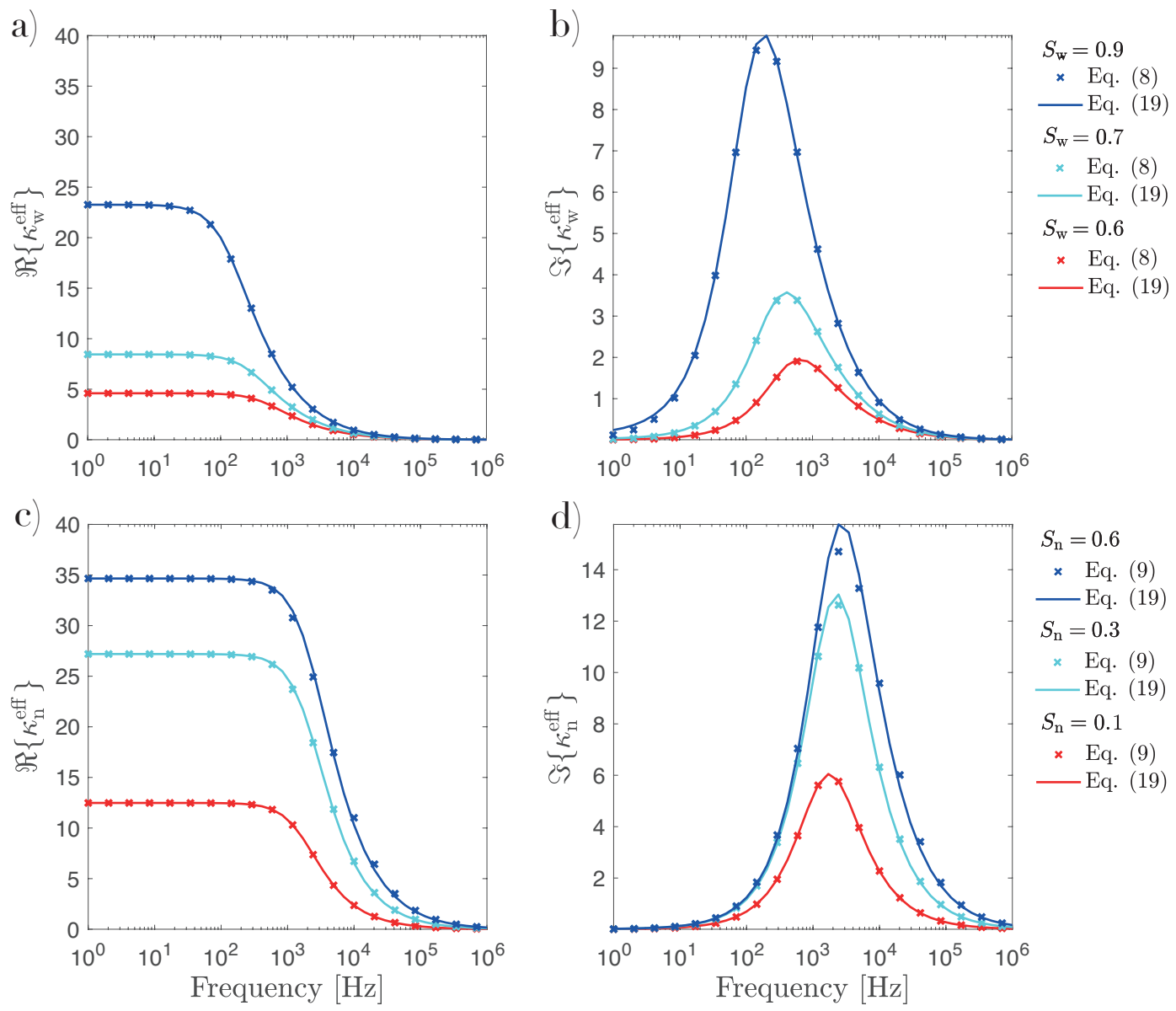

Figure 5. Real and imaginary parts of (a, b) $\kappa_{\mathrm{w}}^{\mathrm{eff}}$ and (c, d) $\kappa_{\mathrm{n}}^{\mathrm{eff}}$ as functions of frequency for different saturation states. The behaviour predicted by eqs (8) and (9) is denoted with crosses, while the response of eq. (19) is represented by solid lines. The corresponding permeability values are expressed in units of Darcy.

progressively narrower pores. These results show that further efforts are needed to develop physically based models of $\Lambda$ in partially saturated porous media.

\section{CONCLUSIONS}

We have presented a physically founded dynamic permeability model for porous media saturated by two immiscible fluid phases. Our results show that the effective permeability functions associated with the wetting and non-wetting pore fluid phases exhibit distinct behaviours with frequency and saturation. Due to the different wetting properties of the pore fluids, the critical frequency decreases with saturation for the wetting phase, while it remains largely unaffected for the non-wetting phase. This finding demonstrates that estimating a dynamic permeability by approximating a partially saturated medium by a fully saturated one using a single pore fluid with effective properties is physically incorrect and error prone. We also analyzed the performance of the frequency scaling of the classical model 

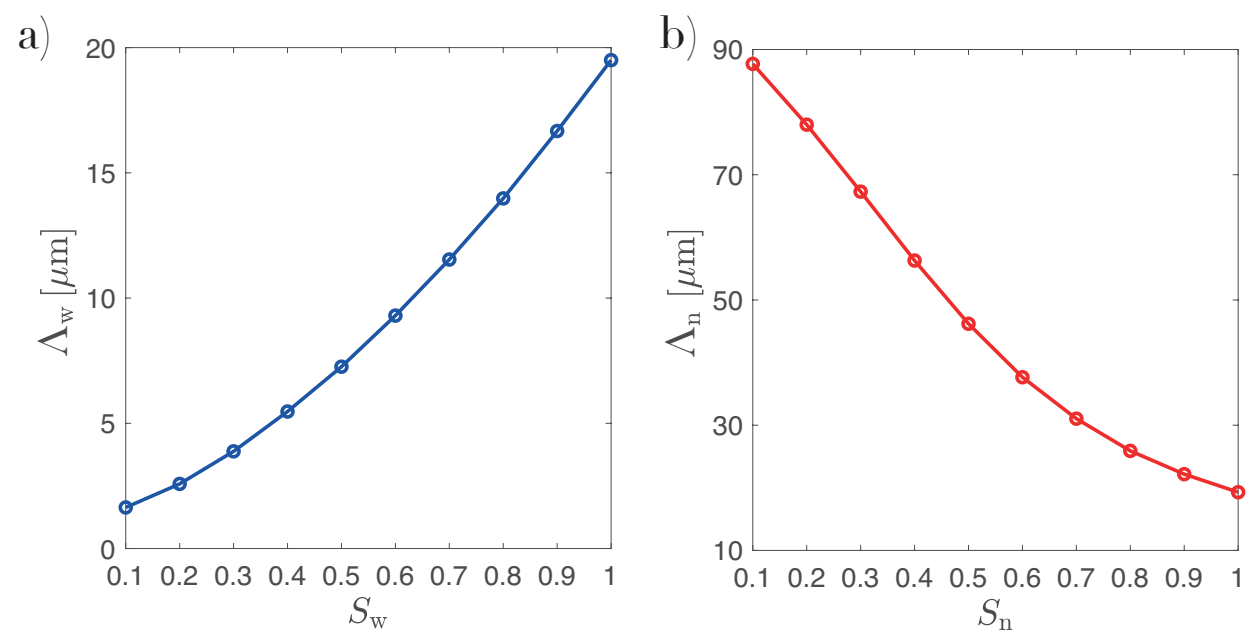

Figure 6. Values of $\Lambda$ as a function of saturation for (a) wetting and (b) non-wetting fluid phases.

of Johnson et al. (1987) in partially saturated scenarios and provide a new definition of the critical frequency in such a context. The dynamic effective permeability functions proposed in this work thus open the perspective of an improved hydraulic characterisation of partially saturated porous media through seismic and seismoelectric methods. This possibility is particularly interesting and pertinent for unconsolidated granular sediments, which are ubiquitous in the vadose zone and for which the transition from viscous- to inertia-dominated flow occurs at rather low frequencies.

\section{ACKNOWLEDGMENTS}

This manuscript greatly benefited from lucid comments and helpful suggestion by the editor-in-chief Jörg Renner and two anonymous reviewers. Part of this work was completed within the Swiss Competence Center for Energy Research-Supply of Electricity with support of Innosuisse. J. G. R. gratefully acknowledges the financial support received from the Agencia Nacional de Promoción Científica y Tecnológica of Argentina (PICT 2017-2976) and from the Swiss National Science Foundation (200020-178946).

\section{REFERENCES}

Achdou, Y. \& Avellaneda, M., 1992. Influence of pore roughness and pore-size dispersion in estimating the permeability of a porous medium from electrical measurements, Phys. Fluids A, 4(12), 2651-2673.

Assouline, S. \& Or, D., 2008. Air entry-based characteristic length for estimation of permeability of variably compacted earth materials, Water Resour. Res., 44(11).

Auriault, J.-L., Borne, L., \& Chambon, R., 1985. Dynamics of porous saturated media, checking of the generalized law of darcy, J. Acoust. Soc. Am., 77(5), 1641-1650. 
Barrière, J., Bordes, C., Brito, D., Sénéchal, P., \& Perroud, H., 2012. Laboratory monitoring of P waves in partially saturated sand, Geophys. J. Int., 191(3), 1152-1170.

Bear, J., 1972. Dynamics of Fluids in Porous Media, Elsevier, N. Y.

Bedford, A., Costley, R., \& Stern, M., 1984. On the drag and virtual mass coefficients in Biot's equations, J. Acoust. Soc. Am., 76(6), 1804-1809.

Bernabé, Y., 1997. The frequency dependence of harmonic fluid flow through networks of cracks and pores, Pure Appl. Geophys., 149(3), 489-506.

Biot, M. A., 1956a. Theory of propagation of elastic waves in a fluid saturated porous solid. I. Low frequency range., J. Acoust. Soc. Am., 28, 168-178.

Biot, M. A., 1956b. Theory of propagation of elastic waves in a fluid saturated porous solid. II. Higher frequency range., J. Acoust. Soc. Am., 28, 179-191.

Blunt, M. J., 2017. Multiphase Flow in Permeable Media: A Pore-Scale Perspective, Cambridge University Press.

Bordes, C., Sénéchal, P., Barrìre, J., Brito, D., Normandin, E., \& Jougnot, D., 2015. Impact of water saturation on seismoelectric transfer functions: a laboratory study of coseismic phenomenon, Geophys. J. Int., 200(3), 1317-1335.

Brakensiek, D. \& Rawls, W., 1992. Comment on "Fractal processes in soil water retention" by Scott W. Tyler and Stephen W. Wheatcraft, Water Resour. Res., 28(2), 601-602.

Brooks, R. \& Corey, A., 1964. Hydraulic Properties of Porous Media, Hydrology Papers No. 3, Colorado State University.

Charlaix, E., Kushnick, A., \& Stokes, J., 1988. Experimental study of dynamic permeability in porous media, Phys. Rev. Lett., 61(14), 1595.

Jardani, A. \& Revil, A., 2015. Seismoelectric couplings in a poroelastic material containing two immiscible fluid phases, Geophys. J. Int., 202(2), 850-870.

Johnson, D. L., Koplik, J., \& Dashen, R., 1987. Theory of dynamic permeability and tortuosity in fluidsaturated porous media, J. Fluid Mech., 176, 379-402.

Jougnot, D., Linde, N., Revil, A., \& Doussan, C., 2012. Derivation of soil-specific streaming potential electrical parameters from hydrodynamic characteristics of partially saturated soils, Vadose Zone J., 11(1).

Jouniaux, L. \& Bordes, C., 2012. Frequency-dependent streaming potentials: A review, Int. J. Geophys., 2012. Kozeny, J., 1927. Über kapillare Leitung des Wassers im Boden, Akad. Wiss, 136(2a), 271-306.

Lenormand, R., 1990. Liquids in porous media, J. Phys. Condens. Matter, 2, 79-88.

Lo, W.-C., Sposito, G., \& Majer, E., 2005. Wave propagation through elastic porous media containing two immiscible fluids, Water Resour. Res., 41(2).

Maineult, A., Jougnot, D., \& Revil, A., 2017. Variations of petrophysical properties and spectral induced polarization in response to drainage and imbibition: a study on a correlated random tube network, Geophys. J. Int., 212(2), 1398-1411.

Mavko, G., Mukerji, T., \& Dvorkin, J., 2009. The Rock Physics Handbook: Tools for Seismic Analysis of 
Porous Media, Cambridge University Press.

Mualem, Y., 1976. A new model for predicting the hydraulic conductivity of unsaturated porous media, Water Resour. Res., 12(3), 513-522.

Pride, S. R., 1994. Governing equations for the coupled electromagnetics and acoustics of porous media, Phys. Rev. B, 50(21), 15678.

Pride, S. R., 2005. Relationships between seismic and hydrological properties, in Hydrogeophysics, chap. 9, pp. 253-290, eds Rubin, Y. \& Hubbard, S., Springer.

Pride, S. R., Morgan, F. D., \& Gangi, A. F., 1993. Drag forces of porous-medium acoustics, Phys. Rev. B, 47(9), 4964.

Schön, J. H., 2015. Physical Properties of Rocks: Fundamentals and Principles of Petrophysics, vol. 65, Elsevier.

Sheng, P. \& Zhou, M.-Y., 1988. Dynamic permeability in porous media, Phys. Rev. Lett., 61(14), 1591.

Smeulders, D., Eggels, R., \& Van Dongen, M., 1992. Dynamic permeability: reformulation of theory and new experimental and numerical data, J. Fluid Mech., 245, 211-227.

Soldi, M., Guarracino, L., \& Jougnot, D., 2017. A simple hysteretic constitutive model for unsaturated flow, Transport Porous Med., 120(2), 271-285.

Vargaftik, N. B., Volkov, B. N., \& Voljak, L. D., 1983. International tables of the surface tension of water, $J$. Phys. Chem. Ref. Data, 12(3), 817-820.

Yu, B., 2008. Analysis of flow in fractal porous media, Appl. Mech. Rev., 61(5), 050801.

Zhou, M.-Y. \& Sheng, P., 1989. First-principles calculations of dynamic permeability in porous media, Phys. Rev. B, 39(16), 12027. 